\title{
Computing Blocking Probabilities in Multi-Class Wavelength Routing Networks *
}

\author{
Sridhar Ramesh George N. Rouskas Harry G. Perros \\ TR-99-08
}

May 24, 1999

\begin{abstract}
We present an approximate analytical method to evaluate efficiently and accurately the blocking performance of wavelength routing networks with multiple classes of calls. The model is fairly general and allows each source-destination pair to service calls of different classes, with each call occupying one wavelength per link. Our approximate analytical approach involves two steps. The arrival process of calls on some routes is first modified slightly to obtain an approximate multi-class network model. Next, all classes of calls on a particular route are aggregated to give an equivalent single-class model. Thus, path decomposition algorithms for single-class wavelength routing networks may be readily extended to the multi-class case. Our work is a first step towards understanding the issues arising in wavelength routing networks that serve multiple classes of customers.
\end{abstract}

Keywords: Wavelength division multiplexing (WDM), Wavelength routing, Multi-class networks, Blocking Probabilities

Department of Computer Science

North Carolina State University

Raleigh, NC 27695-7534

${ }^{*}$ This work was supported by the NSF under grant ANI-9805016. 


\section{Introduction}

A basic property of single mode optical fiber is its enormous low-loss bandwidth of several tens of terahertz $(\mathrm{THz})$. Unfortunately, due to dispersive effects and limitations in optoelectronic component technology, single channel transmission is limited in speed to only a small fraction of the fiber capacity. To take full advantage of the potential of fiber, the use of wavelength division multiplexing (WDM) techniques has become the option of choice [4, 10], and WDM networks have been a subject of research both theoretically [19, 18] and experimentally $[7,8]$. Multiwavelength optical networks have the potential of delivering an aggregate throughput in the order of Terabits per second, and they appear as a viable approach to satisfying the ever-growing demand for more bandwidth per user on a sustained, long-term basis.

The wavelength routing mesh architecture appears promising for Wide Area Network (WAN) distances [6, $22,5]$. The network architecture consists of wavelength routers and fiber links that interconnect them. A wavelength router is capable of switching a light signal at a given wavelength from any input port to any output port. A router may also be capable of enforcing a shift in wavelength [16], in which case a light signal may emerge from the switch at a different wavelength than the one it arrived. By appropriately configuring the routers, all-optical paths (lightpaths) may be established between pairs of nodes in the network. Lightpaths represent direct optical connections without any intermediate electronics. Because of the long propagation delays, and the time required to configure the routers, wavelength routing WANs are expected to operate in circuit-switched mode $[18,6]$. This architecture is attractive for two reasons: first, the same wavelength can be used simultaneously at different parts of the network, and second, the signal power is channeled to the receiver and is not spread to the entire network as in the broadcast-and-select approach [17]. Hence, wavelength routing WANs can be highly scalable.

Given the installed base of optical fiber, and the maturing of optical component technology, it appears that current network technologies are transitory, and will eventually evolve to an all-optical, largely passive infrastructure. Although the long-term result of such an evolution is not yet clear, a feasible scenario for near-term large-scale all-optical networks has emerged in recent years [4, 10]. Wavelength routing WANs are an integral part of this scenario, since it is envisioned that they will act as the backbone that provides the interconnection for local area photonic sub-networks attached to them. The contribution of our work is the development of an approximate, but accurate and efficient, analytical framework for evaluation of the performance of multi-class wavelength routing optical networks.

The problem of computing call blocking probabilities under static (fixed or alternate) routing with random wavelength allocation and with or without wavelength converters has been studied in $[1,14,3$, 11, 19, 21]. The model presented in [1] is based on the assumption that wavelength use on each link is characterized by a fixed probability, independently of other wavelengths and links, and thus, it cannot capture

the dynamic nature of traffic. In [14] it was assumed that statistics of link loads are mutually independent, an approximation that is not accurate for sparse network topologies. The work in [3] developed a Markov chain with state-dependent arrival rates to model call blocking in arbitrary mesh topologies and fixed routing; it was extended in [11] to alternate routing. While more accurate, this approach is computationally intensive and can only be applied to networks of small size in which paths have at most three links. A more tractable 
model was presented in [19] to recursively compute blocking probabilities assuming that the load on link $i$ of a path depends only on the load of link $i-1$. Finally, a study of call blocking under non-Poisson input traffic was presented in [21], under the assumption that link loads are statistically independent.

Other wavelength allocation schemes, as well as dynamic routing are harder to analyze. First-fit wavelength allocation was studied using simulation in [5, 14], and it was shown to perform better than random allocation, while an analytical overflow model for first-fit allocation was developed in [12]. A dynamic routing algorithm that selects the least loaded path-wavelength pair was also studied in [12], and in [15] an unconstrained dynamic routing scheme with a number of wavelength allocation policies was evaluated. Except in $[19,20]$, all other studies assume that either all or none of the wavelength routers have wavelength conversion capabilities. The work in [19] takes a probabilistic approach in modeling wavelength conversion by introducing the converter density, which represents the probability that a node is capable of conversion independently of other nodes in the network. While this approach works well when the objective is the estimation of the expected call blocking performance, it cannot be used to calculate the actual blocking probability on individual paths when the placement of converters is known, nor can it be used to compare various converter placement strategies. Finally, in [20] a dynamic programming algorithm to determine the location of converters on a single path that minimizes average or maximum blocking probability was developed under the assumption of independent link loads.

Most of the approximate analytical techniques developed for computing blocking probabilities in wavelength routing networks $[14,3,11,21,12,15,20]$ make the assumption that link blocking events are independent and amount to the well-known link decomposition approach [9], while the development of some techniques is based on the additional assumption that link loads are also independent. Link decomposition has been extensively used in conventional circuit-switched networks where there is no requirement for the same wavelength to be used on successive links of the path taken by a call. The accuracy of these underlying approximations also depends on the traffic load, the network topology, and the routing and wavelength allocation schemes employed. While link decomposition techniques make it possible to study the qualitative behavior of wavelength routing networks, more accurate analytical tools are needed to efficiently evaluate the performance of these networks efficiently, as well as to tackle complex network design problems, such as selecting the optical switches where to employ wavelength converters.

Our group has considered the problem of computing call blocking probabilities in mesh wavelength routing networks with fixed and alternate routing and random wavelength allocation. Unlike previous studies, we have developed an iterative path decomposition algorithm [9] for analyzing arbitrary network topologies. Specifically, we analyze a given network by decomposing it into a number of path sub-systems. The latter are analyzed in isolation using our previous results on blocking in a single path in a wavelength routing network [23]. The individual solutions are appropriately combined to form a solution for the overall network, and the process repeats until the blocking probabilities converge. Our approach accounts for the correlation of both link loads and link blocking events, giving accurate results for a wide range of loads and network topologies. It also allows non-uniform traffic, in the sense that call request arrival rates vary for each sourcedestination pair. Our algorithm can compute call blocking probabilities in a mesh network where only a 


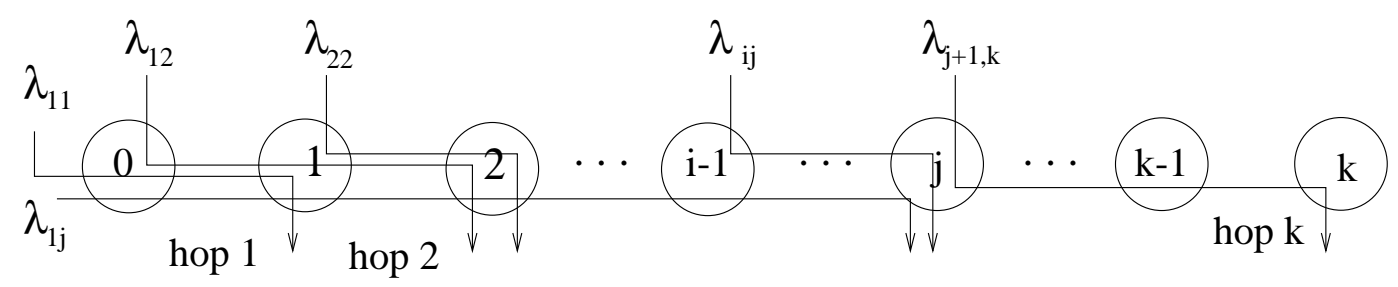

Figure 1: A $k$-hop path

fixed but arbitrary subset of nodes are capable of wavelength conversion. Therefore, our algorithm can be an important tool in the development and evaluation of converter placement strategies. Finally, in [25] we studied the first-fit and most-used wavelength allocation policies, and we showed that they have almost identical performance in terms of blocking probability for all calls in the network. We also demonstrated that the blocking probabilities under the random wavelength allocation policy with no converters and with converters at all nodes provide upper and lower bounds for the values of the blocking probabilities under the first-fit and most-used policies.

All previous studies of blocking probabilities discussed here have only considered single-class wavelength routing networks. It is expected, however, that future applications will be utilized by a wide range of applications with varying characteristics in terms of their arrival rates and call holding times. In this paper, we present a method to extend the results in $[23,24]$ to multi-class optical networks. While multi-class circuit-switched networks have been studied in the literature [2], to the best of our knowledge, this is the first time that multi-class wavelength routing networks are analyzed.

The development of our approximate analytical techniques involves two steps. The arrival process of calls on some routes is first modified slightly to obtain a modified multi-class network model. Next, all classes of calls on a particular route are aggregated to give an equivalent single-class model. This equivalent single-class model has the same call blocking probability on any given route as the modified multi-class network, and can be easily solved due to the existence of a product-form solution. Simulation results further show that these results approximate the blocking probability of the original multi-class network with good accuracy. Thus, solutions for single-class networks developed in [23, 24] may be extended to the multi-class case.

In Section 2, we describe the multi-class wavelength routing network under study. In Section 3, we explain how the modified multi-class model and the equivalent single-class model are obtained. Section 4 contains the numerical results involving comparisons between performance measures obtained from the approximate model and from the exact model through simulations. We conclude the paper in Section 6

\section{The Multi-Class Wavelength Routing Network}

We consider a wavelength routing network with an arbitrary topology. Each link in the network supports exactly $W$ wavelengths, and each node is capable of transmitting and receiving on any of these $W$ wavelengths. Call requests between a source and a destination node arrive at the source according to a Poisson 
process with a rate that depends on the source-destination pair. If the request can be satisfied, an optical circuit is established between the source and destination for the duration of the call. Further, calls between any two nodes may be of several classes. Without any loss of generality, we may assume that there are $R$ classes of calls. Call holding times are assumed to be exponentially distributed, with a mean that depends on the class of the call.

In our model, we allow some of the nodes in the network to employ wavelength converters. These nodes can switch an incoming wavelength to an arbitrary outgoing wavelength. (When there are converters at all nodes, the situation is identical to that in classical circuit-switching networks, a special case of the more general scenario discussed here.) If no wavelength converters are employed in the path between the source and the destination, a call can only be established if the same wavelength is free on all the links used by the call. This is known as the wavelength continuity requirement, and it increases the probability of call blocking. If a call cannot be established due to lack of available wavelengths, the call is blocked. On the other hand, if a call can be accommodated, it is randomly assigned one of the wavelengths that are available on the links used by the call ${ }^{1}$. Thus, we only consider the random wavelength assignment policy in this paper.

Since many of our results are developed in terms of a single path in a wavelength routing network, we now introduce the relevant notation. A $k$-hop path, such as the one shown in Figure 1, consists of $k+1$ nodes. Call these Node 0 , Node $1, \cdots$, Node $k$. Nodes $(i-1)$ and $i, 1 \leq i \leq k$, are said to be connected by Link $i$. A segment is a sub-path that includes links $i$ through $j, j \geq i \geq 1$. Calls originating at node $i-1$ and terminating at node $j$ use the above segment, which we shall denote by the pair $(i, j)$. Calls between these two nodes may belong to one of $R$ classes. These calls are also be said to use route $(i, j)$. We also define the following parameters.

- $\lambda_{i j}^{(r)}, j \geq i, 1 \leq r \leq R$, is the Poisson arrival rate of calls of Class $r$ that originate at node $(i-1)$ and terminate at node $j$.

- $1 / \mu_{i j}^{(r)}$ is the mean of the exponentially distributed service time of calls of Class $r$ that originate at node $(i-1)$ and terminate at node $i$. We also let $\rho_{i j}^{(r)}=\lambda_{i j}^{(r)} / \mu_{i j}^{(r)}$.

- $N_{i j}^{(r)}(t)$ is the number of active calls at time $t$ on segment $(i, j)$ belonging to Class $r$.

- $F_{i j}(t)$ is the number of wavelengths that are free on all hops of segment $(i, j)$ at time $t$. A call that arrives at time $t$ and uses route $(i, j)$ is blocked if $F_{i j}(t)=0$.

\footnotetext{
${ }^{1}$ In a path with wavelength converters, a wavelength is randomly assigned within each segment of the path whose starting and ending nodes are equipped with converters.
} 


\section{Blocking Probabilities in A Single Path of a Network}

\subsection{The Single-Class Case}

We briefly review our previous results for a path of a single-class wavelength routing network. Consider the $k$-hop path shown in Figure 1. Let the state of this system at time $t$ be described by the $k^{2}$-dimensional process:

$$
\underline{X}_{k}(t)=\left(N_{11}(t), N_{12}(t), \cdots, N_{k k}(t), F_{12}(t), F_{13}(t), \cdots, F_{1 k}(t), F_{23}(t), \cdots, F_{(k-1) k}(t)\right)
$$

A closer examination of the process $\underline{X}_{k}(t)$ reveals that it is not time-reversible (see [23]). This result is true in general, when $k \geq 2$ and $W \geq 2$.

Since the number of states of process $\underline{X}_{k}(t)$ grows very fast with the number $k$ of hops in the path and the number $W$ of wavelengths, it is not possible to obtain the call blocking probabilities directly from the above process. Consequently, an approximate model was constructed in [23] to analyze a single-class, $k$-hop path of a wavelength routing network. The approximation consists of modifying the call arrival process to obtain a time-reversible Markov process that has a closed-form solution. To illustrate our approach, let us consider the Markov process corresponding to a 2-hop path:

$$
\underline{X}_{2}(t)=\left(N_{11}(t), N_{12}(t), N_{22}, F_{12}(t)\right)
$$

We now modify the arrival process of calls that use both hops (a Poisson process with rate $\lambda_{12}$ in the exact model) to a state-dependent Poisson process with rate $\Lambda_{12}$ given by:

$$
\Lambda_{12}\left(n_{11}, n_{12}, n_{22}, f_{12}\right)=\lambda_{12} \frac{f_{12}\left(W-n_{12}\right)}{f_{11} f_{12}}
$$

The arrival process of other calls remain as in the original model. As a result, we obtain a new Markov process $\underline{X}_{2}^{\prime}(t)$ with the same state space and the same state transitions as process $\underline{X}_{2}(t)$, but which differs from the latter in some of the state transition rates.

We made the observation in [23] that under the new arrival process (3) for calls using both hops, the Markov process $\underline{X}_{2}^{\prime}(t)$ is time-reversible and the invariant $\pi$ is given by:

$$
\pi\left(n_{11}, n_{12}, n_{22}, f_{12}\right)=\frac{1}{G_{2}(W)}\left(\frac{\rho_{11}^{n_{11}}}{n_{11} !} \frac{\rho_{12}^{n_{12}}}{n_{12} !} \frac{\rho_{22}^{n_{22}}}{n_{22} !}\right) \times \frac{\left(\begin{array}{c}
f_{11} \\
f_{12}
\end{array}\right)\left(\begin{array}{c}
n_{11} \\
W-n_{12}-n_{22}-f_{12}
\end{array}\right)}{\left(\begin{array}{c}
W-n_{12} \\
W-n_{12}-n_{22}
\end{array}\right)}
$$

where $G_{k}(W)$ is the normalizing constant for a $k$-hop path with $W$ wavelengths.

Let $P\left(n_{11}, n_{12}, n_{22}\right)$ be the marginal distribution over the states for which $N_{i j}(t)=n_{i j}, 1 \leq i \leq j \leq 2$. It can be verified [23] that

$$
P\left(n_{11}, n_{12}, n_{22}\right)=\frac{1}{G_{2}(W)}\left(\frac{\rho_{11}^{n_{11}}}{n_{11} !} \frac{\rho_{12}^{n_{12}}}{n_{12} !} \frac{\rho_{22}^{n_{22}}}{n_{22} !}\right)
$$


Likewise, for a $k$-hop path, $k \geq 2$, with the modified state-dependent Poisson arrival process, the marginal distribution over the states for which $N_{i j}(t)=n_{i j}, 1 \leq i \leq j \leq k$, is given by:

$$
P\left(n_{11}, n_{12}, \cdots, n_{k k}\right)=\frac{1}{G_{k}(W)}\left(\prod_{\{(i, j) \mid 1 \leq i \leq j \leq k\}} \frac{\rho_{i j}^{n_{i j}}}{n_{i j} !}\right)
$$

It is easily seen that this distribution is the same as in the case of a network with wavelength converters at each node. An interesting feature of having wavelength converters at every node is that the network has a product-form solution even when there are multiple classes of calls on each route, as long as call arrivals are Poisson, and holding times are exponential [13,9]. Further, when calls of all classes occupy the same number of wavelengths, we can aggregate classes to get an equivalent single class model with the same steady-state probability distribution over the aggregated states, as we show next.

\subsection{The Multi-Class Case}

Let us now consider a $k$-hop path with wavelength converters at all nodes, and with at $R$ classes of calls. If $\lambda_{i j}^{(r)}, 1 \leq i \leq j \leq k, 1 \leq r \leq R$, is the arrival rate of calls of Class $r$ on route $(i, j)$, and $1 / \mu_{i j}^{(r)}, 1 \leq i \leq j \leq k$, $1 \leq r \leq R$, is the mean of the exponential holding time of calls of Class $r$, the probability of being in state $\underline{n}=\left(n_{11}^{(1)}, n_{11}^{(2)}, \cdots, n_{11}^{(R)}, n_{12}^{(1)}, \cdots, \cdots, n_{k k}^{(R)}\right)$ is given by:

$$
P\left(n_{11}^{(1)}, n_{11}^{(2)}, \cdots, n_{11}^{(R)}, n_{12}^{(1)}, \cdots, n_{12}^{(R)}, \cdots, n_{k k}^{(R)}\right)=\frac{1}{G_{k}(W)}\left(\prod_{\{(i, j) \mid 1 \leq i \leq j \leq k\}} \prod_{r=1}^{R} \frac{\left(\rho_{i j}^{(r)}\right)^{n_{i j}^{(r)}}}{n_{i j}^{(r)} !}\right)
$$

Let $\sigma_{i j}=\sum_{r} \rho_{i j}^{(r)}$ and $s_{i j}=\sum_{r} n_{i j}^{(r)}=s_{i j}$. As defined, $s_{i j}$ is the total number of calls of all classes that use segment $(i, j)$ of the path, and $\sigma_{i j}$ is the total offered load of these calls. Taking the summation of (7) over all states such that $\sum_{r} n_{i j}^{(r)}=s_{i j}, 1 \leq i \leq j \leq r$, we obtain:

$$
P^{\prime}\left(s_{11}, s_{12}, \cdots, s_{k k}\right)=\sum_{\left\{\underline{n} \mid \sum_{n} n_{i j}^{(r)}=s_{i j}\right\}} P(\underline{n})=\frac{1}{G_{k}(W)}\left(\prod_{\{(i, j) \mid 1 \leq i \leq j \leq k\}} \frac{\sigma_{i j}^{s_{i j}}}{s_{i j} !}\right)
$$

Observe that this is identical to the solution (6) for the single-class case obtained by substituting $\sigma_{i j}$ by $\rho_{i j}$ and $s_{i j}$ by $n_{i j}$ in (8).

Based on the above results, we conclude that by employing class aggregation on a multi-class path with converters at all nodes, we obtain a system equivalent to a single-class path with converters. In Section 3.1, we showed that the modified single-class wavelength routing network without converters has a steady-state marginal distribution similar to the exact single-class network with converters. We now show that a modified multi-class network without wavelength converters can also be subjected to class aggregation that results in an equivalent single-class model. The modification applied to the arrival process of calls is similar to the single-class case, and it is given by:

$$
\Lambda_{i j}^{(r)}(\underline{x})=\lambda_{i j}^{(r)} \frac{\left.f_{i j}\left(\sum_{l=1}^{i} s_{l i}+f_{i i}\right)\left(\sum_{l=1}^{i+1} s_{l(i+1)}+f_{(i+1)(i+1)}\right) \cdots \sum_{l=1}^{j-1} s_{l(j-1)}+f_{(m-1)(m-1)}\right)}{f_{i i} f_{(i+1)(i+1)} \cdots f_{j j}}
$$


Then, the probability that the equivalent single class network without converters is in state

$$
S_{\underline{x}}=\left(s_{11}, s_{12}, \cdots, s_{1 k}, s_{22}, \cdots, s_{k k}, f_{12}, f_{13}, \cdots, f_{(k-1) k}\right)
$$

is given by:

$$
\pi\left(S_{\underline{x}}\right)=\left(\prod_{i, j} \frac{s_{i j} !}{\sigma_{i j}^{s_{i j}}}\right)\left(\prod_{l=2}^{k} \frac{\left(\begin{array}{c}
f_{1(l-1)} \\
f_{1 l}
\end{array}\right)\left\{\prod_{m=2}^{l-1}\left(\begin{array}{c}
f_{m(l-1)}-f_{(m-1)(l-1)} \\
f_{m l}-f_{(m-1) l}
\end{array}\right)\right\}\left(\begin{array}{c}
f_{l l}+n_{l l}-f_{(l-1)(l-1)} \\
f_{l l}-f_{(l-1) l}
\end{array}\right)}{\left(\begin{array}{c}
f_{l l}+n_{l l} \\
f_{l l}
\end{array}\right)}\right)
$$

Once again, the parameters of the single-class model are given by:

$$
\begin{gathered}
s_{i j}=\sum_{r=1}^{R} n_{i j}^{(r)} \quad 1 \leq i<j \leq k \\
\sigma_{i j}=\sum_{r=1}^{R} \rho_{i j}^{(r)} \quad 1 \leq i<j \leq k
\end{gathered}
$$

\subsection{Blocking Probabilities in the Multi-Class Case}

Since the arrival rate of calls of each class on each route is Poisson, the blocking probability of a call of Class $r$ using route $(i, j)$ is given by:

$$
Q_{i j}=\lim _{\tau \rightarrow \infty} \frac{\int_{t=0}^{\tau} I_{\left\{F_{i j}(\tau)=0\right\}} d t}{\tau}
$$

As can be seen, the blocking probability is class-independent. Further, the modified arrival process satisfies the criterion:

$$
\frac{\Lambda_{i j}^{(r 1)}(\underline{x})}{\Lambda_{i j}^{(r 2)}(\underline{x})}=\frac{\lambda_{i j}^{(r 1)}}{\lambda_{i j}^{(r 2)}} \quad 1 \leq r 1, r 2 \leq r
$$

Therefore, the blocking probability for Class $r$ on route $(i, j)$ on the modified process, given by:

$$
Q_{i j}^{\prime}=\frac{\sum_{\left\{\underline{x} \mid f_{i j}=0\right\}} \Lambda_{i j}^{(r)}(\underline{x}) \pi(\underline{x})}{\sum_{\underline{x}} \Lambda_{i j}^{(r)}(\underline{x}) \pi(\underline{x})}
$$

can also be seen to be independent of the Class $r$. Thus, by computing the blocking probability on the equivalent single-class path, we can obtain the solution to the multi-class path.

\section{Blocking Probabilities in Long Paths and Mesh Topologies}

The solution to single-class wavelength routing networks involving paths with a large number of hops, and for networks with mesh topologies, with wavelength converters at an arbitrary subset of nodes, has been 
discussed in [23] and [24]. These solutions involve decomposition of the network into short path segments with two or three hops, and analyzing these approximately using expressions similar to (4). The solutions to individual segments are appropriately combined to obtain a value for the blocking probability of calls that traverse more than one segment. The effect of the wavelength continuity requirement is captured by an approximate continuity factor that increases the blocking probability of calls continuing to the next segment to account for the possible lack of common free wavelengths in the two segments. The process repeats until the blocking probabilities converge. By applying the transformations given in (12) and (13), the same algorithms may be used to calculate blocking probabilities for multi-class networks.

Specifically, we follow the following steps to compute the blocking probabilities of a wavelength routing network with $R$ classes of calls.

1. Path decomposition: Decompose the multi-class mesh network topology into $L$ single-path subsystems using the algorithm in [24].

2. Time-reversible process approximation: For each single-path sub-system, modify the arrival process as shown in expression (9) to obtain an approximate time-reversible Markov process for the path.

3. Class aggregation: For each sub-system, apply the transformations in (12) and (13) to obtain an equivalent single-class path sub-system.

4. For each path sub-system, obtain the blocking probabilities as follows. If the path is at most three hops long, use expressions (16) and (11) directly. If the path sub-system is longer than three hops, analyze it by decomposing it into 2- or 3-hop paths which are solved in isolation, and combine the individual solutions to obtain the blocking probabilities along the original longer path (see [23]).

5. Repeat Steps 2 to 4, after appropriately modifying the original arrival rates to each single-path subsystem to account for the new values of the blocking probabilities obtained in Step 3 (see [24]), until the blocking probabilities converge within a certain tolerance.

\section{$5 \quad$ Numerical Results}

In this section, we validate the approximate method described in Section 4 by comparing the blocking probabilities for each route as obtained from the approximate method with those obtained through simulation of the exact model.

\subsection{A Single Path of a Network}

We first provide results for 2-hop and 3-hop paths, since these systems represent the basic blocks of our decomposition algorithm. In Table I we show the arrival and service rates for calls on each route $(i, j), 1 \leq$ 
Table 1: Arrival and service parameters for a 2-hop path

\begin{tabular}{|c|c|c|c|c|c|c|}
\hline \hline \multirow{2}{*}{$\begin{array}{c}\text { Route } \\
(i, j)\end{array}$} & \multicolumn{2}{|c|}{ Class 1 } & \multicolumn{2}{c|}{ Class 2} & \multicolumn{2}{c|}{ Class 3 } \\
\cline { 2 - 7 } & $\lambda$ & $\mu$ & $\lambda$ & $\mu$ & $\lambda$ & $\mu$ \\
\hline \hline$(1,1)$ & 3.0 & 8.0 & 4.0 & 8.0 & 3.0 & 6.0 \\
$(1,2)$ & 3.0 & 4.0 & 4.0 & 5.0 & 3.0 & 6.0 \\
$(2,2)$ & 5.0 & 8.0 & 5.0 & 5.0 & 3.0 & 6.0 \\
\hline
\end{tabular}

$j \leq 2$, of a 2-hop path. There are $R=3$ classes of calls for each route. In Figure 2 we plot the blocking probability of calls along each of the three possible routes (recall that blocking probabilities are classindependent) against the number $W$ of wavelengths supported by the links of the path. As we can see, the blocking probability decreases as $W$ increases, as expected. We also observe that calls on Route $(1,2)$ (i.e., calls using both hops of the path) experience the highest blocking probability, since they have to compete against calls using either the first or the second hop of the path. Also, calls on Route $(2,2)$ (that is, those using the second hop only) experience higher blocking probability than those using Route $(1,1)$, a direct consequence of the fact that the offered load of calls on Route $(2,2)$ is higher than that of calls on Route $(1,1)$ (refer to Table 1). Most importantly, however, we can see that there is good agreement between the values of the blocking probabilities obtained through our analytical technique and those obtained through simulation. Similar results have been obtained for different values for the arrival and service parameters and for different number of classes, indicating that our approximate method is accurate over a wide range of network characteristics.

We now consider a 3-hop path with $R=3$ classes of calls, and the arrival and service rates shown in Table 2. In Figure 3, we plot the blocking probability against the number $W$ of wavelengths for three (out of the six) types of calls in this path: calls using Route $(1,1)$, i.e., the first hop of the path, calls on Route $(2,3)$, that is, those using the last two hops, and calls on Route $(1,3)$ using all three hops of the path. The behavior of the blocking probability curves in Figure 3 is similar to that in Figure 2. Specifically, the blocking probability decreases as $W$ increases, while it increases with the number of hops a call must traverse. We also note that the curves derived analytically closely track those obtained by simulation.

From Figures 2 and 3 we conclude that for 2-hop and 3-hop multi-class paths (the building blocks of our decomposition algorithm), the class aggregation and the time-reversible Markov process (derived through the modification (9) of the arrival process) can be used to compute call blocking probabilities accurately. In the next subsection we demonstrate that the same is true for mesh topologies.

\subsection{Mesh Topologies}

In this section we provide results for two different mesh networks: a $3 \times 3$ regular torus topology, and the NSFNET irregular topology. 


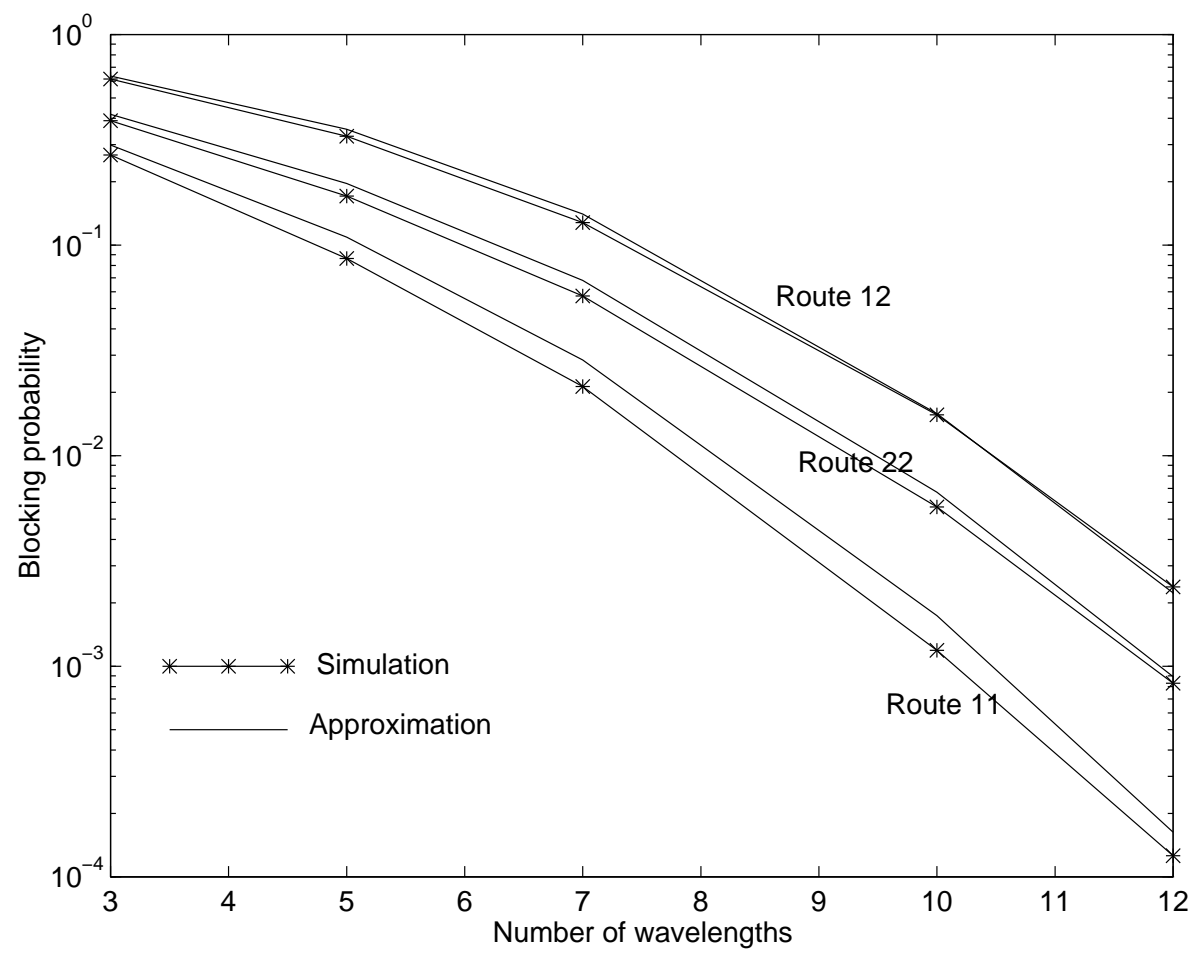

Figure 2: Blocking probabilities for a 2-hop path and the parameters shown in Table 1

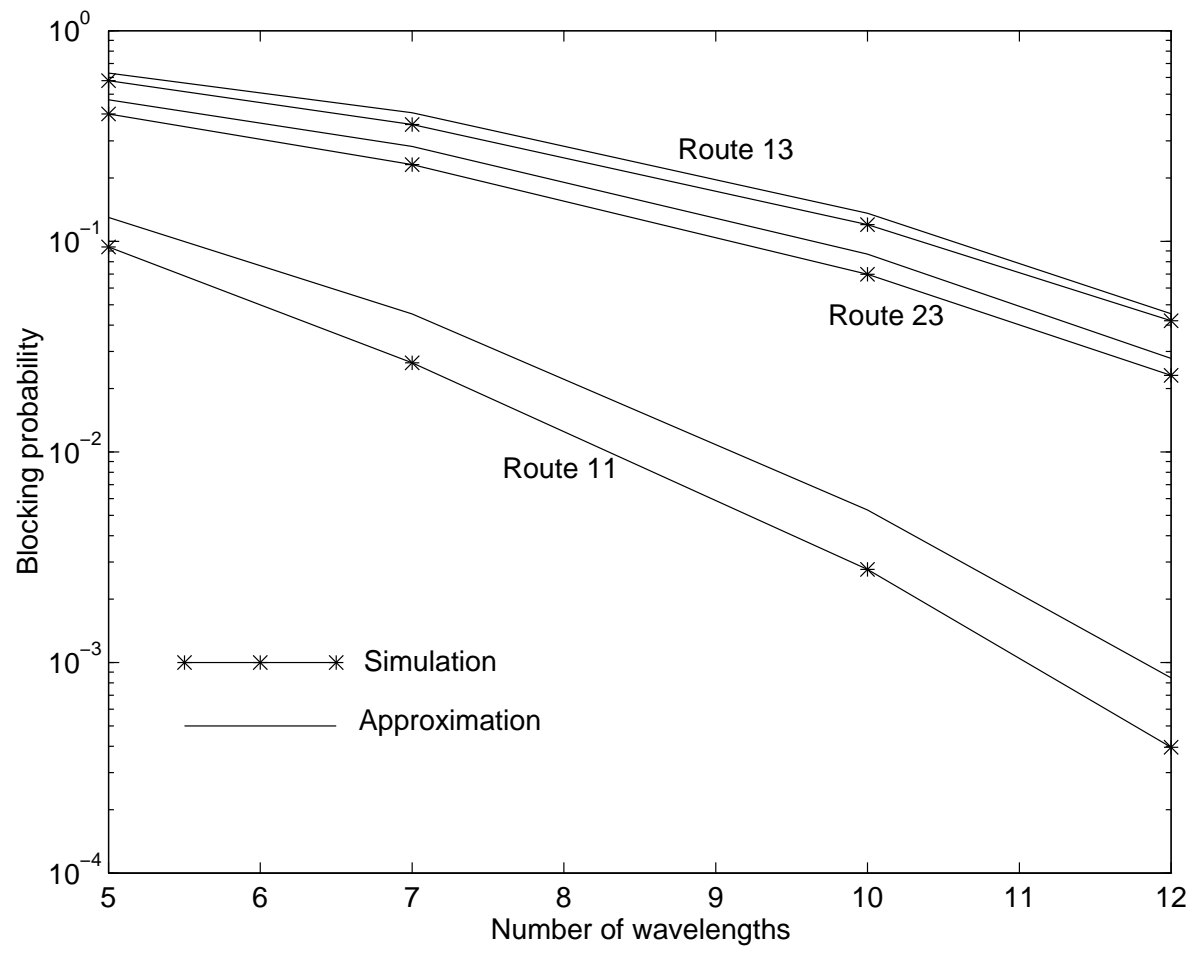

Figure 3: Blocking probabilities for a 3-hop path and the parameters shown in Table 2 
Table 2: Arrival and service parameters for a 3-hop path

\begin{tabular}{|c|c|c|c|c|c|c|}
\hline \hline \multirow{2}{*}{$\begin{array}{c}\text { Route } \\
(i, j)\end{array}$} & \multicolumn{2}{|c|}{ Class 1} & \multicolumn{2}{c|}{ Class 2} & \multicolumn{2}{c|}{ Class 3} \\
\cline { 2 - 7 } & $\lambda$ & $\mu$ & $\lambda$ & $\mu$ & $\lambda$ & $\mu$ \\
\hline \hline$(1,1)$ & 3.0 & 3.0 & 1.0 & 3.0 & 1.0 & 3.0 \\
$(1,2)$ & 1.0 & 6.0 & 2.0 & 6.0 & 1.0 & 6.0 \\
$(1,3)$ & 1.0 & 2.0 & 1.0 & 2.0 & 2.0 & 2.0 \\
$(2,2)$ & 1.0 & 3.0 & 1.0 & 3.0 & 2.0 & 3.0 \\
$(2,3)$ & 1.0 & 2.0 & 1.0 & 2.0 & 3.0 & 2.0 \\
$(3,3)$ & 3.0 & 6.0 & 1.0 & 6.0 & 1.0 & 6.0 \\
\hline
\end{tabular}

Table 3: Arrival and service parameters for the $3 \times 3$ mesh

\begin{tabular}{|c|c|c|c|}
\hline \hline & Class 1 & Class 2 & Class 3 \\
\hline \hline$\lambda$ & 0.4 & 0.6 & 0.5 \\
$\mu$ & 3.0 & 5.0 & 6.0 \\
\hline
\end{tabular}

Let us first consider the $3 \times 3$ network shown in Figure 4 . Each of the nine nodes is directly linked with 4 others to form a toroid interconnection pattern. There are 72 source-destination pairs in the network, and 36 bi-directional routes. Each route requires at most 2 hops. Routes 1 through 18 comprise single-hop paths, while routes 19 through 36 consist of 2-hop paths, as in Figure 4. Once again we study this network with 3 classes of calls. The arrival and service rates of calls of a particular class on each route were assumed identical, and are given in Table 3. We study the blocking probability as a function of the number of wavelengths on each link for two routes: Route 1 (the single-hop path from Node 1 to Node 2) and Route 23 (the 2-hop path from Node 2 to Node 4 ). The approximation and simulation results are plotted in Figure 5, and we observe that they are in good agreement.

Next, we consider a network with topology similar to the NSF Network, shown in Figure 6. There are 16 nodes, and 240 uni-directional routes. There are three classes of calls on each route. The arrival and service rates of calls of a particular class are the same on each route, and are shown in Table 4. The blocking probabilities are plotted in Figure 7 for four routes, as a function of the number of wavelengths on each link. Route A is a single-hop route from nodes 1 to 5 . Route B has two hops, connecting node 1 to node 3 via node 2. Route $\mathrm{C}$ has three hops, connecting node 1 to node 4 via nodes 2 and 3 . Route $\mathrm{D}$ has four hops, connecting node 4 to node 5 via nodes 3,2 and 1 .

From Figure 7 we can see that the length of the path used by a call considerably affects the blocking probability experienced by the call, an observation that is consistent with all our previous results in this section. Specifically, for a given number $W$ of wavelengths, the blocking probability increases with the number 


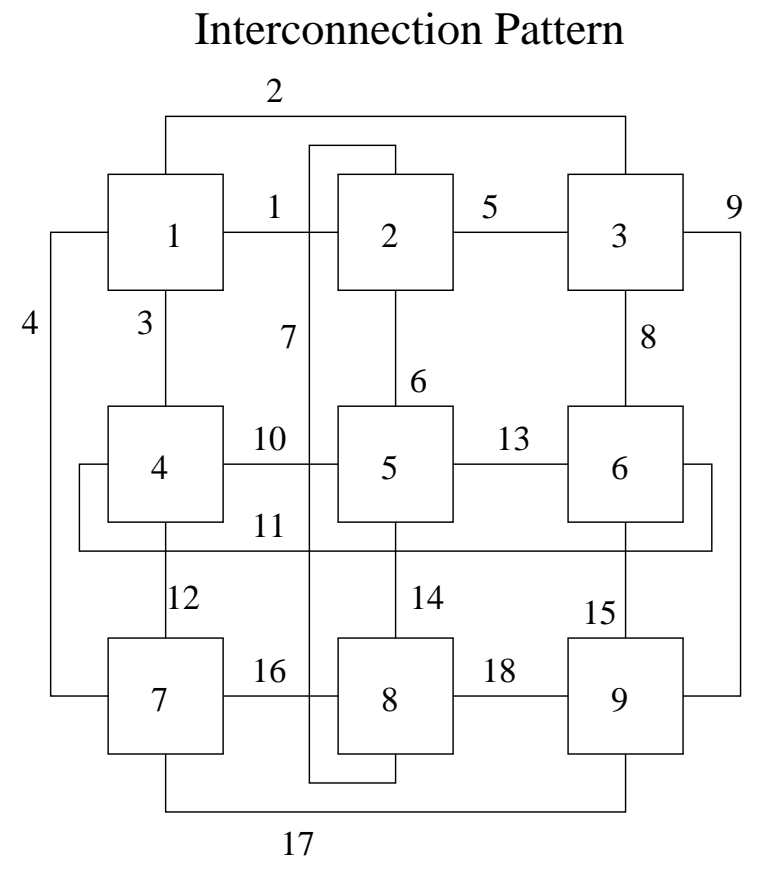

$$
\begin{aligned}
& 2 \text {-hop routes } \\
& \hline 19->1+6 \\
& 20->2+8 \\
& 21->1+7 \\
& 22->2+15 \\
& 23->1+3 \\
& 24->5+8 \\
& 25->1+4 \\
& 26->5+9 \\
& 27->2+3 \\
& 28->5+6 \\
& 29->2+4 \\
& 30->5+7 \\
& 31->10+14 \\
& 32->11+15 \\
& 33->10+12 \\
& 34->13+15 \\
& 35->11+12 \\
& 36->13+14
\end{aligned}
$$

Figure 4: The $3 \times 3$ torus network

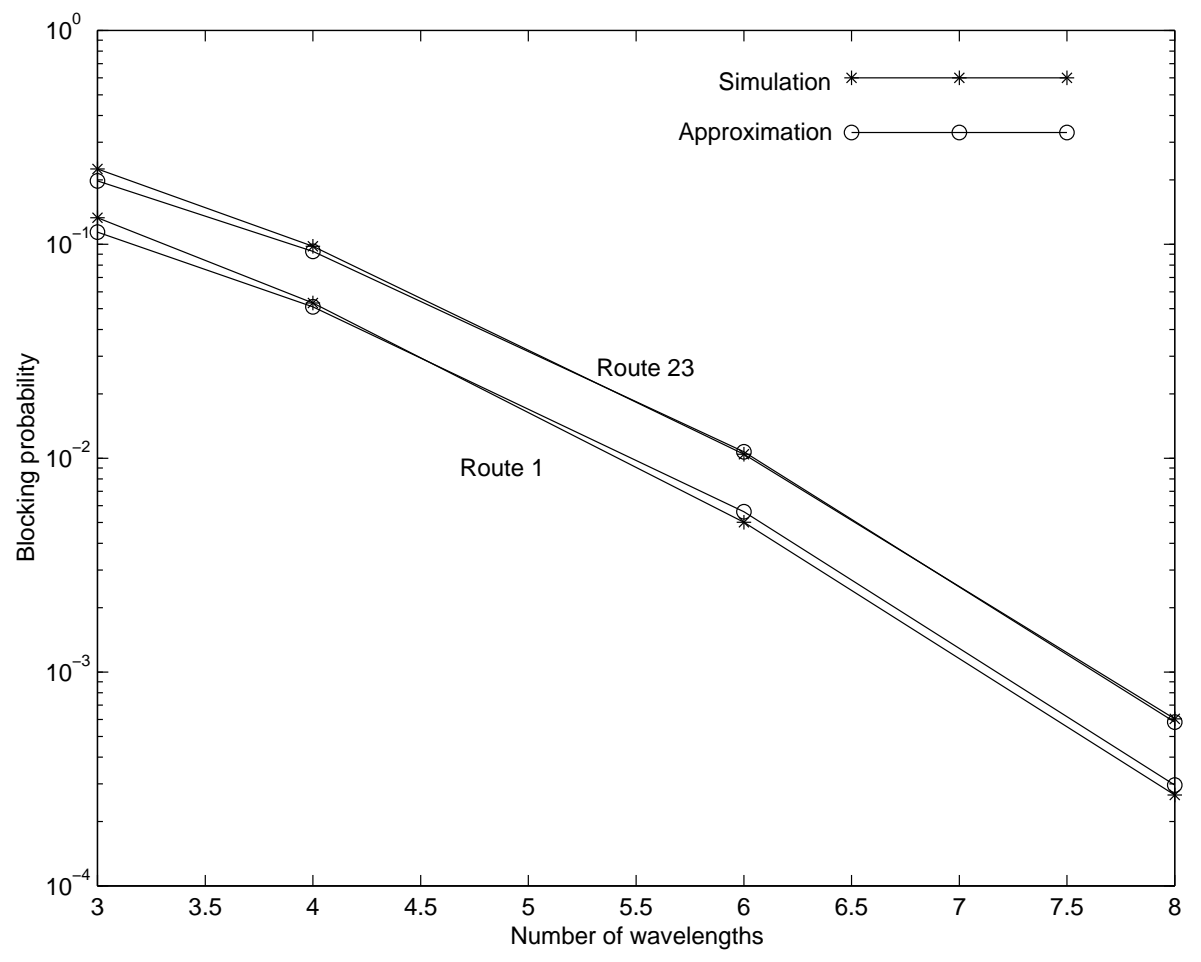

Figure 5: Blocking probabilities for the $3 \times 3$ torus network 
Table 4: Arrival and service parameters for the NSF Network

\begin{tabular}{|c|c|c|c|}
\hline \hline & Class 1 & Class 2 & Class 3 \\
\hline \hline$\lambda$ & 0.1 & 0.2 & 0.15 \\
$\mu$ & 3.0 & 6.0 & 4.0 \\
\hline
\end{tabular}

of hops in a route, such that calls on Route A (a single-hop path) have the lowest blocking probability while calls on Route D (a four-hop path) the highest. Further, as in all previous topologies, the results indicate that our approximation method can be used to estimate accurately the blocking probabilities for all calls in the network.

Results similar to the ones presented in Figures 2-7 have been obtained for a wide range of traffic loads and different classes of calls. Our main conclusion is that our approximate analytical technique can be applied to compute the call blocking probabilities in wavelength routing networks of realistic size and topology.

\section{Concluding Remarks}

We have considered the problem of computing call blocking probabilities in multi-class wavelength routing networks which employ the random wavelength allocation policy. Our approach consists of modifying the call arrival process to obtain an approximate multi-class network model, using class aggregation to map this to an equivalent single-class network, and employing path decomposition algorithms on the latter to determine the call blocking probabilities (which are class-independent). Our work is a first step towards realizing wavelength routing networks that can serve multiple classes of customers. 


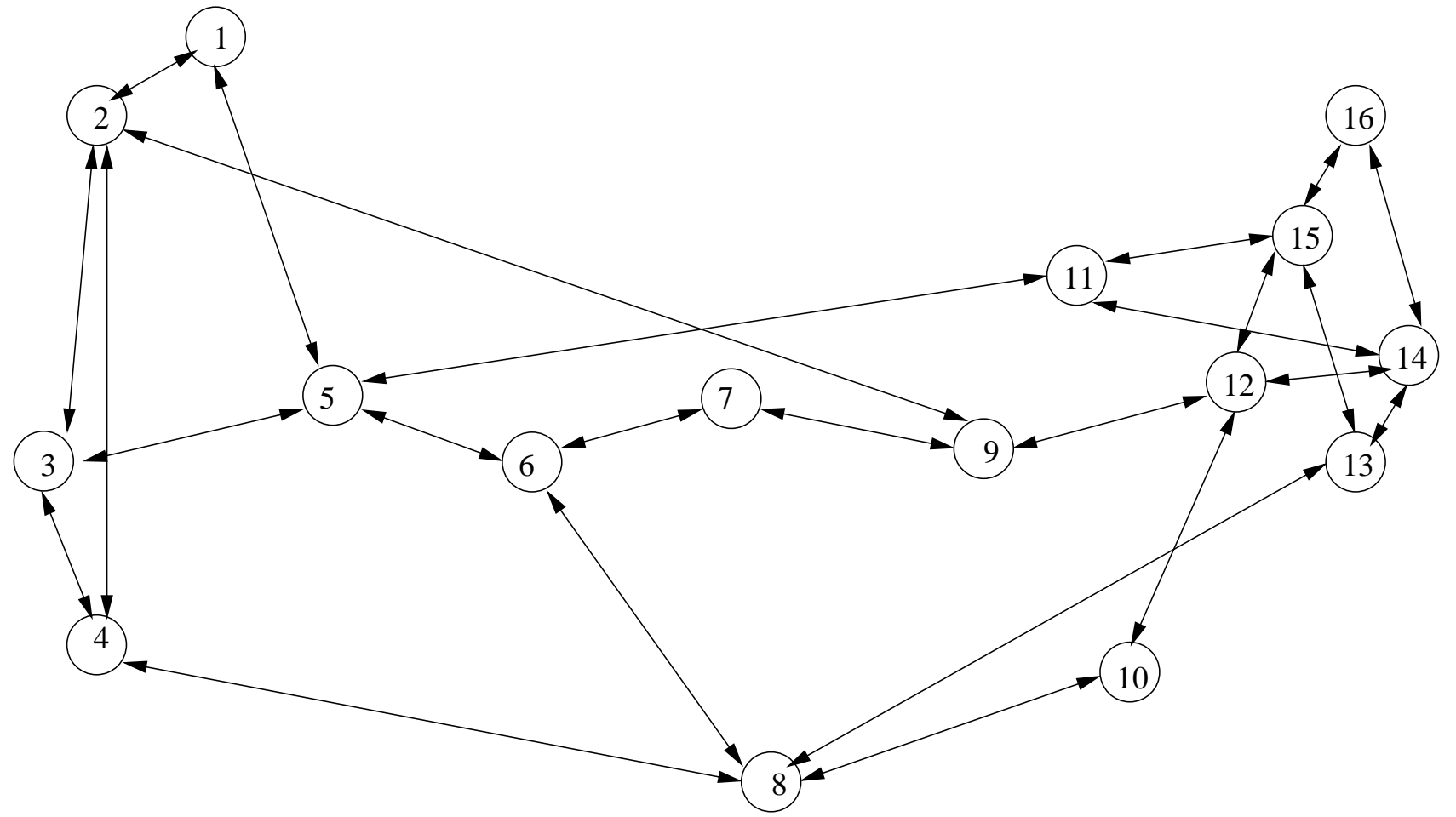

Figure 6: The NSF network

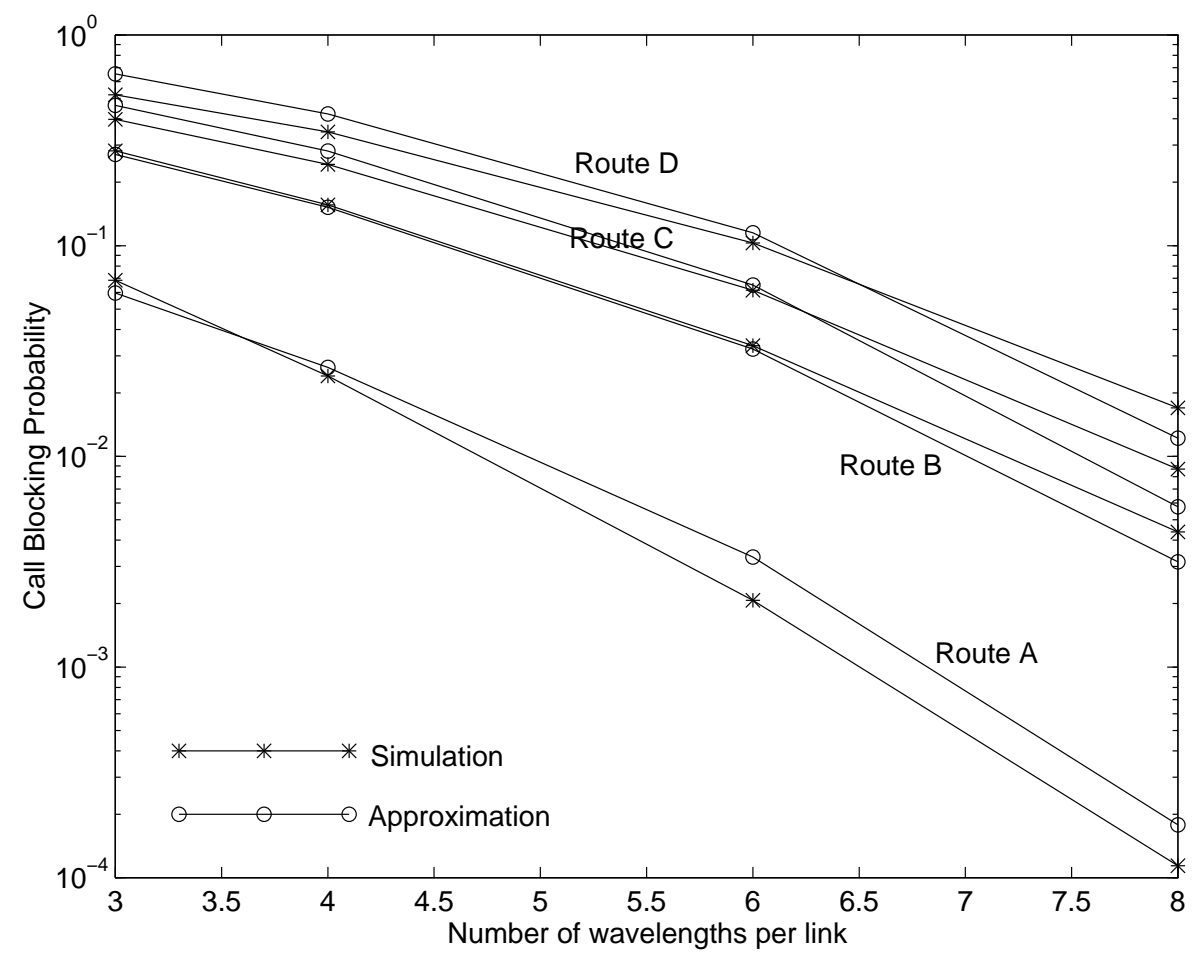

Figure 7: Blocking probabilities for the NSF network 


\section{References}

[1] R. A. Barry and P. A. Humblet. Models of blocking probability in all-optical networks with and without wavelength changers. IEEE Journal Selected Areas in Communications, 14(5):858-867, June 1996.

[2] B. Baynat and Y. Dallery. A product-form approximation method for general closed queueing networks with several classes of customers. Performance Evaluation, 24:165-188, 1993.

[3] A. Birman. Computing approximate blocking probabilities for a class of all-optical networks. IEEE Journal Selected Areas in Communications, 14(5):852-857, June 1996.

[4] C. A. Brackett. Is there an emerging consensus on WDM networking? Journal of Lightwave Technology, 14(6):936-941, June 1996.

[5] I. Chlamtac, A. Ganz, and G. Karmi. Lightpath communications: An approach to high bandwidth optical WANS. IEEE Transactions on Communications, 40(7):1171-1182, July 1992.

[6] B. Mukherjee et al. Some principles for designing a wide-area WDM optical network. IEEE/ACM Transactions on Networking, 4(5):684-696, October 1996.

[7] E. Hall et al. The Rainbow-II gigabit optical network. IEEE Journal Selected Areas in Communications, 14(5):814-823, June 1996.

[8] R. E. Wagner et al. MONET: Multiwavelength optical networking. Journal of Lightwave Technology, 14(6):1349-1355, June 1996.

[9] A. Girard. Routing and Dimensioning in Circuit-Switched Networks. Addison Wesley, Reading, MA, 1990.

[10] P. E. Green. Optical networking update. IEEE Journal Selected Areas in Communications, 14(5):764779, June 1996.

[11] H. Harai, M. Murata, and H. Miyahara. Performance of alternate routing methods in all-optical switching networks. In Proceedings of INFOCOM '97, pages 517-525. IEEE, April 1997.

[12] E. Karasan and E. Ayanoglu. Effects of wavelength routing and selection algorithms on wavelength conversion gain in wdm optical networks. IEEE/ACM Transactions on Networking, 6(2):186-196, April 1998.

[13] F. P. Kelly. Reversibility and Stochastic Networks. John Wiley \& Sons, New York, 1979.

[14] M. Kovacevic and A. Acampora. Benefits of wavelength translation in all-optical clear-channel networks. IEEE Journal Selected Areas in Communications, 14(5):868-880, June 1996.

[15] A. Mokhtar and M. Azizoglu. Adaptive wavelength routing in all-optical netowrks. IEEE/ACM Transactions on Networking, 6(2):197-206, April 1998. 
[16] B. Ramamurty and B. Mukherjee. Wavelength conversion in WDM networking. IEEE Journal Selected Areas in Communications, 16(7):1061-1073, Septemer 1998.

[17] R. Ramaswami. Multiwavelength lightwave networks for computer communication. IEEE Communications Magazine, pages 78-88, February 1993.

[18] R. Ramaswami and K. N. Sivarajan. Design of logical topologies for wavelength-routed optical networks. IEEE Journal Selected Areas in Communications, 14(5):840-851, June 1996.

[19] S. Subramaniam, M. Azizoglu, and A. Somani. All-optical networks with sparse wavelength conversion. IEEE/ACM Transactions on Networking, 4(4):544-557, August 1996.

[20] S. Subramaniam, M. Azizoglu, and A. K. Somani. On the optimal placement of wavelength converters in wavelength-routed networks. In Proceedings of INFOCOM '98, pages 902-909. IEEE, April 1998.

[21] S. Subramanian, A. K. Somani, M. Azizoglu, and R. A. Barry. A performance model for wavelength conversion with non-poisson traffic. In Proceedings of INFOCOM '97, pages 500-507. IEEE, April 1997.

[22] N. Wauters and P. Demeester. Design of the optical path layer in multiwavelength cross-connected network. IEEE Journal Selected Areas in Communications, 14(5):881-892, June 1996.

[23] Y. Zhu, G. N. Rouskas, and H. G. Perros. Blocking in wavelength routing networks, Part I: The single path case. In Proceedings of INFOCOM '99, pages 321-328. IEEE, March 1999.

[24] Y. Zhu, G. N. Rouskas, and H. G. Perros. Blocking in wavelength routing networks, Part II: Mesh topologies. In Proceedings of the Sixteenth International Teletraffic Congress (ITC 16). Elsevier Science, June 1999.

[25] Y. Zhu, G. N. Rouskas, and H. G. Perros. Bounds on the blocking performance of allocation policies in wavelength routing networks and a study of the effects of converters. Technical Report TR-99-01, North Carolina State University, Raleigh, NC, January 1999. 